\title{
Angiogenic changes in co-cultures of mast cells and myocardial microvascular endothelial cells under hyperglycemic conditions
}

\author{
ZHIHUA WANG $^{1,2^{*}}$, QINGYONG ZHANG $^{1 *}$, WEI ZHU ${ }^{1}$, JIANPING TAO $^{1}$ and MENG WEI ${ }^{1}$ \\ ${ }^{1}$ Division of Cardiology, Shanghai Jiaotong University Sixth Hospital, Shanghai 200233; \\ ${ }^{2}$ Division of Cardiology, Yancheng First Hospital, Yancheng 224000, P.R. China
}

Received December 6, 2012; Accepted February 18, 2013

DOI: $10.3892 /$ ijmm.2013.1293

\begin{abstract}
The aim of the present study was to determine the correlation between angiogenesis and the differential expression of growth factors and their receptors when myocardial microvascular endothelial cells (MMVECs) were co-cultured with mast cell granules (MCGs) under hyperglycemic conditions. MMVECs and mast cells (MCs) were isolated from Wistar rats. An in vitro angiogenesis assay was used to observe any differences when MMVECs were co-cultured with MCGs in normal or hyperglycemic medium. The mRNA and protein expression of growth factors and their receptors were analyzed by real-time reverse transcription (RT)-PCR and western blot analysis. Real-time RT-PCR analysis demonstrated the upregulated mRNA and protein expression of vascular endothelial growth factor (VEGF) in the MMVECs; however, the expression of its receptor, fms-like tyrosine kinase-1 (Flt-1) and fetal liver kinase-1 (Flk-1), decreased significantly, and the angiogenic ability of the MMVECs decreased under hyperglycemic conditions. The angiogenic ability of the MMVECs cultured under hyperglycemic conditions (even after the addition of MCGs) was inferior to that of the MMVECs cultured under normal glucose conditions. The specific inhibitor of tryptase, N-tosyl-L-lysine chloromethyl ketone (TLCK), suppressed angiogenesis regardless of the glucose concentration, and the specific inhibitor of chymase, N-tosyl-L-phenylalanyl chloromethyl ketone (TPCK), was not as effective as TLCK, which was mainly detected under hyperglycemic conditions. High glucose levels have a profound effect on angiogenesis; this effect may be more pronounced than the effects of MCGs on angiogenesis.
\end{abstract}

Correspondence to: Dr Meng Wei, Division of Cardiology, Shanghai Jiaotong University Sixth Hospital, 600 Yishan Road, Shanghai 200233, P.R. China

E-mail: weim.sjtu6h@hotmail.com

${ }^{*}$ Contributed equally

Key words: microvascular endothelial cells, mast cell, hyperglycemia, angiogenesis

\section{Introduction}

Mast cell (MC) functions have classically been associated with allergic responses (1), with previous studies indicating that these cells contribute to other common diseases, such as atherosclerosis, aortic aneurysm and cancer (2-4). Liu et al (5) found that MCs also contribute to obesity and diabetes. Mechanism studies have demonstrated that MCs contribute to white adipose tissue (WAT) and muscle angiogenesis. Aside from supplying WAT with nutrients, microvessels also provide a path for leukocyte infiltration followed by adipokine release $(6,7)$. Reduced angiogenesis should limit nutrient supply, thereby impairing cell viability $(8,9)$, and the inhibition of angiogenesis blocks adipose tissue development in mice.

Angiogenesis is a regulated balance between stimulatory and inhibitory factors. This process is regulated by several pro-angiogenic factors, such as vascular endothelial growth factor (VEGF), angiopoietin-1 (Ang-1), fibroblast growth factor-2 [(FGF-2), also known as basic fibroblast growth factor (bFGF)], as well as anti-angiogenic factors, such as thrombospondin-1, angiostatin and Ang-2. Among various angiogenic factors, angiogenesis is mainly regulated by the interplay between VEGF and angiopoietins $(10,11)$. A number of studies have shown that VEGF and angiopoietins are associated with diabetes in angiogenesis $(12,13)$. However, angiogenesis in diabetes, which is associated with the dysregulation of neovascularization, has also been recognized. The molecular defects underlying these angiogenic abnormalities have generated much interest but remain elusive.

Microvascular endothelial cells are commonly used to study the mechanisms of diabetic complications, since they play an essential role in the abnormal angiogenesis process of several diseases, including diabetes mellitus. In the present study, we established a co-culture system to investigate the correlation between angiogenesis and various growth factors in vitro.

\section{Materials and methods}

Reagents. All cell culture reagents, alamarBlue ${ }^{\circledR}$ cell viability reagent, TRIzol reagent, the SuperScript ${ }^{\mathrm{TM}}$ III First-Strand Synthesis System for reverse transcription (RT)-PCR and Platinum SYBR-Green qPCR SuperMix were purchased from 
Invitrogen Life Technologies, Inc. (Grand Island, NY). Primary antibodies against VEGF, fms-like tyrosine kinase-1 (Flt-1), fetal liver kinase-1 (Flk-1), $\beta$-actin and secondary antibodies against either rabbit or mouse IgG were purchased from Santa Cruz Biotechnology, Inc. (Santa Cruz, CA). SuperSignal West Pico Chemiluminescent Substrate was obtained from Pierce Biotechnology, Inc. (Rockford, IL). All other reagents were obtained from Sigma-Aldrich Chemical Co. (St. Louis, MO).

Cell culture of myocardial microvascular endothelial cells (MMVECS). Wistar male rats [8-9 weeks of age; Shanghai Laboratory Animal Center (SLAC), Shanghai Institutes for Biological Science, Shanghai, China] were used for primary MMVEC isolation. The Animal Care Committee of Shanghai Jiaotong University approved the animal protocols. MMVECs were isolated as previously described (14). Briefly, the rats were anesthetized with sodium pentobarbital $(60 \mathrm{mg} / \mathrm{kg})$. After thoracotomy, the hearts were rapidly removed and washed in phosphate-buffered saline (PBS). The atria, visible connective tissue, valvular tissue, the right ventricle, and the epicardial and endocardial surfaces of the left ventricle were carefully removed, and the remaining myocardial tissue was cut into sections $\left(1 \mathrm{~mm}^{3}\right)$. Myocardial tissues were then seeded on culture plates pre-coated with rat-tail tendon gelatin and incubated at $37^{\circ} \mathrm{C}$ in a humidified atmosphere of $5 \% \mathrm{CO}_{2} / 95 \%$ air. After a 40-min attachment period, the tissues were cultured in DMEM (25 mmol/l D-glucose) supplemented with $20 \%$ fetal bovine serum (FBS), $50 \mathrm{U} / \mathrm{ml}$ heparin, $100 \mathrm{U} / \mathrm{ml}$ penicillin and $100 \mathrm{~g} / \mathrm{ml}$ streptomycin. The tissue pieces were discarded and the medium was changed after approximately $70 \mathrm{~h}$. MMVECs were identified by their typical 'cobblestone' appearance with positive CD31 and CD34 immunostaining. Only MMVECs at second passage were used for experiments; cells were allowed to grow to $80-90 \%$ confluence followed by serum-starvation for $24 \mathrm{~h}$ before the experiments commenced.

Collection and isolation of MCs. MCs were isolated as previously described, with minor modifications to the procedure (15). Briefly, MCs from the peritoneal cavities of male Wistar rats (14-16 weeks of age; SLAC, Shanghai Institutes for Biological Science) were collected by lavage with $15 \mathrm{ml}$ of RPMI-1640 (1\% FBS). After a 1-h attachment period in the incubator, nonadherent cells, mainly MCs, were separated by using percoll density gradient centrifugation at $2,500 \mathrm{rpm}$ for $15 \mathrm{~min}$ at $4^{\circ} \mathrm{C}$. Cells that remained at the percoll interface were aspirated and re-suspended in PBS. MCs were washed in PBS twice and cultured in RPMI-1640, supplemented with $10 \%$ FBS, $25 \mathrm{mM}$ HEPES, $100 \mathrm{U} / \mathrm{ml}$ penicillin and $100 \mathrm{mg} / \mathrm{ml}$ streptomycin. MCs isolated by this procedure exceeded $90 \%$ purity based on staining with $0.05 \%$ toluidine blue $\mathrm{O}$.

Preparation of MC granules (MCGs). The standard incubation was conducted in $200 \mu \mathrm{l}$ of RPMI- 1640 containing $5.0 \times 10^{6} \mathrm{cell} /$ $\mathrm{ml} \mathrm{MCs}$. After the MCs were pre-incubated at $37^{\circ} \mathrm{C}$ for $15 \mathrm{~min}$, compound 48/80 (5 $\mu \mathrm{g} / \mathrm{ml})$, a non-cytotoxic MC-specific stimulator, was added to stimulate the MCs for $1 \mathrm{~min}$. The MCs were centrifuged at $800 \mathrm{xg}$ for $5 \mathrm{~min}$ and the supernatant containing the material released from the stimulated MCs was stored at $-80^{\circ} \mathrm{C}$ for further experiments (16). The tryptase and chymase activity of the MCGs was measured before each experiment, using the same method as previously described (17). In principle, tryptase and chymase activities were quantified using the chromogenic substrates, $N \alpha$-benzoyl-DL-arginine- $p$-nitroanilide hydrochloride (BAPNA) and $N$-succinyl-L-phenylalanine$p$-nitroanilide (SAAPP), respectively. Tryptase activity was determined by its ability to cleave a synthetic substrate, BAPNA ( $2 \mathrm{mmol} / \mathrm{l})$ in Tris- $\mathrm{HCl}(0.1 \mathrm{~mol} / \mathrm{l} ; \mathrm{pH} 8.0)$ and glycerol $(1 \mathrm{~mol} / \mathrm{l})$ at $410 \mathrm{~nm}$, while chymase activity was determined spectrophotometrically by the rate of hydrolysis of SAAPP $(0.7 \mathrm{mmol} / \mathrm{l})$ in $\mathrm{NaCl}(1.5 \mathrm{~mol} / \mathrm{l})$ and Tris $(0.3 \mathrm{~mol} / \mathrm{l} ; \mathrm{pH} 8.0)$ at $405 \mathrm{~nm}$. Protease activity was expressed in milliunits/millilitre $(\mathrm{mU} / \mathrm{ml})$, in which 1 unit of enzyme activity was defined as the $1 \mu \mathrm{mol}$ of degraded substrate $/ \mathrm{min}$ at $25^{\circ} \mathrm{C}$. The tryptase activity of the MCGs used in the present study was $9.46 \mathrm{mU} / \mathrm{ml}$, and the chymase activity was $3.57 \mathrm{mU} / \mathrm{ml}$.

MMVEC-MC co-culture. MMVECs $\left(1 \times 10^{5}\right.$ cells/well) and MCGs obtained from $1 \times 10^{5}$ activated MCs were co-cultured in DMEM with low $(5.56 \mathrm{mmol} / \mathrm{l})$ and high glucose levels ( $25 \mathrm{mmol} / \mathrm{l})$. The inhibitors of tryptase [N-tosyl-L-lysine chloromethyl ketone (TLCK)] and chymase [N-tosyl-L-phenylalanyl chloromethyl ketone (TPCK)] were used in further experiments when MMVECs were grown to $85-90 \%$ confluence before being quiesced in DMEM with $1 \%$ FBS for $24 \mathrm{~h}$ and treated with MCGs.

Cell proliferation assay. The cell proliferation rate was assessed by cell counting using alamarBlue ${ }^{\circledR}$ cell viability reagent. Second passage MMVECs were seeded onto 96-well cell culture plates at an initial density of $5 \times 10^{3}$ cells/well $(100 \mu \mathrm{l})$ in DMEM with 5\% FBS in the presence or absence of MCGs. Following overnight incubation, $10 \mu \mathrm{l}$ of alamarBlue ${ }^{\circledR}$ cell solution were added and further incubated for $2 \mathrm{~h}$. Cell growth rate was quantified by the difference in absorbance at 570 and $600 \mathrm{~nm}$ using a microplate reader (Tecan Group Ltd., Männedorf, Switzerland), and the absorbance reading was repeated for 5 consecutive days to generate a cell growth curve.

Cell migration assay. Cell migration was evaluated using a transwell chamber assay (Corning Inc., Corning, NY). In brief, second passage MMVECs were trypsinized, resuspended in $200 \mu 1$ serum-free DMEM $\left(4 \times 10^{4}\right.$ cells/well $)$ and were then placed onto the upper chamber, while another $800 \mu \mathrm{l}$ of DMEM with a different glucose concentration and $2 \%$ FBS were placed in the lower compartment of the transwell chamber. MCGs, TLCK or TPCK were also added to examine their effects on cell migration activity, as described above. Following incubation for $12 \mathrm{~h}$ at $37^{\circ} \mathrm{C}$, allowing time for the cells to migrate from the upper to the lower chamber, cells that migrated to the lower side of the transwell inserts were washed, fixed with methanol for $10 \mathrm{~min}$ and then stained with crystal violet dye for cell counting. Cell migration ability was quantified by an average of cells in 5 random microscopic fields (x200 magnification) at the lower side of the transwell insert.

Cell scratch wound healing assay. Wound healing assay was performed as described previously (18). Once second passage MMVECs reached approximately $90 \%$ confluence in 24-well plates, a wound was made with a $200 \mu$ l pipette tip. The cells were washed with PBS and further incubated in DMEM with a 
different glucose concentration and $1 \%$ FBS in the presence or absence of MCGs. The effects of TLCK and TPCK on MCGs were also examined. After $24 \mathrm{~h}$ of incubation, cultured cells were photographed and cell migration activity was quantified with ImageJ software (version 1.37) by measuring the area of the cells that moved beyond a reference line.

Capillary-like tube formation assay on Matrigel. Matrigel (BD Biosciences, Bedford, MA) was used for the capillary-like tube formation assay. Briefly, $100 \mu \mathrm{l} /$ well of this matrix solution were added to a 24 -well culture plate and $30 \mathrm{~min}$ were allowed for gelation at $37^{\circ} \mathrm{C}$. MMVECs obtained as described above, which had been resuspended in DMEM with a different glucose concentration and $1 \%$ FBS, were added to the top of the Matrigel $\left(5 \times 10^{4}\right.$ cells/well) and incubated for $18 \mathrm{~h}$ at $37^{\circ} \mathrm{C}$. Tube formation was carefully observed either in the presence or absence of MCGs, and the effects of TLCK and TPCK were also examined. Once cell culture images were captured, tube structures were quantified by counting all branches in 3 random fields from each well (19). Each experiment was repeated 3 times.

Real-time RT-PCR. Total RNA was extracted from MMVECs using TRIzol Reagent according to the manufacturer's instructions. RNA $(2 \mu \mathrm{g})$ was then reverse-transcribed into cDNA using the SuperScript ${ }^{\mathrm{TM}}$ III First-strand Synthesis System for RT-PCR and was further amplified by SYBR-Green RT-PCR amplification with specific oligonucleotides as follows: VEGF sense, 5'-GCA CTG GAC CCT GGC TTT AC-3' and antisense primer, 5'-CTG CAG GAA GCT CAT CTC TC-3'; Flk-1 sense, 5'-ACA GTT CCC AGA GTG GTT GG-3' and antisense primer, 5'-GTC ACT GAC AGA GGC GAT GA-3'; Flt-1 sense, 5'-CCA CTT CTG TCT TGC CAC ACA-3' and antisense primer, 5'-CCA ACC AAT TAA GAC CTT CTG-3'; and $\beta$-actin sense, 5'-CAC CCG CGA GTA CAA CCT TC-3' and antisense primer, 5'-CCC ATA CCC ACC ATC ACA CC-3'. The SYBR-Green RT-PCR amplification was carried out in a $25 \mu \mathrm{l}$ reaction volume that contained $12.5 \mu \mathrm{l} 2 \mathrm{X}$ Platinum SYBR-Green qPCR SuperMix, $200 \mathrm{nM}$ each of the forward and reverse primer, and $2 \mu \mathrm{l}$ of diluted cDNA using the iCycler $\mathrm{iQ}^{\mathrm{TM}}$ Real-Time PCR Detection System (Bio-Rad, Richmond, CA). The thermal profile for SYBR-Green real-time RT-PCR was conducted for 40 cycles under the following conditions: denaturation at $94^{\circ} \mathrm{C}$ for $30 \mathrm{sec}$, annealing at optimal temperature for each primer pair for $30 \mathrm{sec}$, and extension at $72^{\circ} \mathrm{C}$ for $45 \mathrm{sec}$. Final extension was at $72^{\circ} \mathrm{C}$ for $10 \mathrm{~min}$. A single amplification product was confirmed by running a melting curve for all PCRs. Each sample was assayed in triplicate.

Western blot analysis for VEGF, Flt-1 and Flk-1. MMVECs were lysed in lysis buffer, and the protein content was measured using the BCA assay (both from Beyotime, China). Equal amounts of protein were loaded and separated onto an 8 to $12 \%$ Tris-glycine gel and transferred onto PVDF membranes. Primary antibodies against VEGF (1:500 dilution), Flt-1 (1:500 dilution), Flk-1 (1:500 dilution) and $\beta$-actin (1:500 dilution) were added to the membranes, incubated overnight at $4^{\circ} \mathrm{C}$, followed by reaction with appropriate secondary antibodies (1:4,000 dilution, goat anti-rabbit IgG and goat anti-mouse IgG-HRP linked; Cell Signaling Technology) for $2 \mathrm{~h}$. ECL reagent was then used to determine the immunoreaction, which was measured by densitometry on X-ray film, using GIS software (Bio-Tanon, Shanghai, China). All western blot analysis experiments were repeated at least 3 times with different cell preparations.

Statistical analysis. Data are presented as the means \pm SD. Statistical significance was assessed by one-way ANOVA followed by post hoc analysis using the Student-NewmanKeuls test. A P-value $<0.05$ was considered to indicate a statistically significant difference. Statistical analyses were performed using SPSS 16.0 statistical software (SPSS Inc., Chicago, IL).

\section{Results}

Impaired angiogenesis of MMVECs in high glucose. MMVEC proliferation, migration and capillary-like tube formation are important components of the process of angiogenesis. The proliferation, migration and capillary-like tube formation ability of the MMVECS co-cultured with MCGs increased significantly in spite of high or low glucose. However, the MMVECs cultured under low glucose conditions showed a higher proliferation rate compared with those co-cultured with MCGs under hyperglycemic conditions from day 3 to 5 (Fig. 1) $(\mathrm{P}<0.05)$. This phenomenon was also observed in both cell scratch wound healing assays and the capillary-like tube formation of MMVECs cultured under low glucose conditions compared to those co-cultured with MCGs under hyperglycemic conditions (Fig. 1). The effect of MCGs on MMVECs was significantly inhibited by the addition of TLCK and TPCK, resulting in even lower migration ability and capillary-like tube formation $(\mathrm{P}<0.05$, respectively). There was no significant difference observed between low and high glucose conditions when TLCK was added to the MMVECs co-cultured with MCGs. When TPCK was added to the MMVECs co-cultured with MCGs under low glucose conditions, there was a significant increase in migration ability and capillary-like tube formation compared to the MMVECs co-cultured with MCGs under high glucose conditions (Fig. 2) $(\mathrm{P}<0.05)$.

Analysis of the mRNA expression changes by qRT-PCR. VEGF mRNA expression increased in a time-dependent manner in the MMVECs co-cultured with MCGs within $2 \mathrm{~h}$, with the peak occurring after $4 \mathrm{~h}$ of culture in hyperglycemic medium and after $8 \mathrm{~h}$ of culture in low-glucose medium $(\mathrm{P}<0.05)$. VEGF mRNA expression in the MMVECs cultured under hyperglycemic conditions was significantly higher compared to that in MMVECs cultured under low glucose conditions, in spite of the MCGs, and this increase continued for up to $24 \mathrm{~h}$ (Fig. 3A). Flt-1 mRNA expression in the MMVECs decreased in a time-dependent manner, with a peak present after $4 \mathrm{~h}$ of culture under hyperglycemic conditions and after $8 \mathrm{~h}$ of culture under low glucose conditions $(\mathrm{P}<0.05)$. Flt-1 mRNA expression in the MMVECs co-cultured with MCGs was significantly higher than that in MMVECs cultured in different concentrations of glucose. Flt-1 mRNA expression in the MMVECs co-cultured with MCGs under hyperglycemic conditions was significantly lower compared to that in the MMVECs cultured under low glucose conditions and this 
A

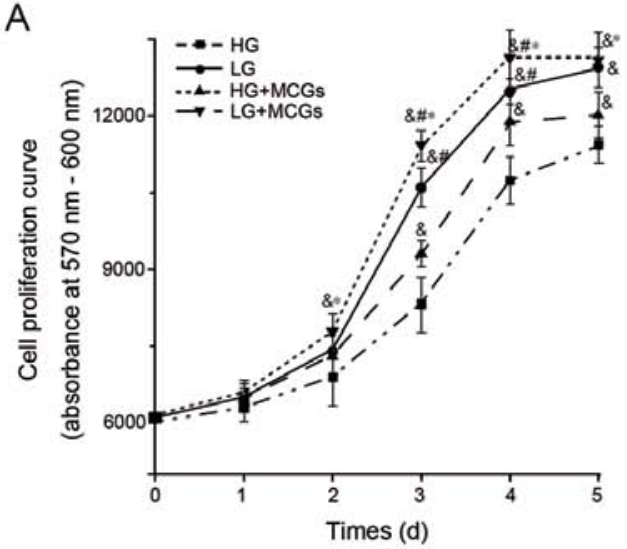

B

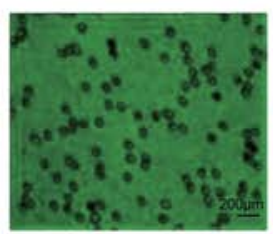

LG

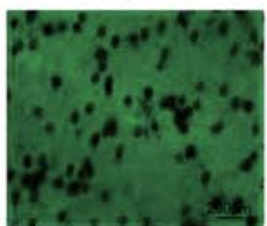

HG

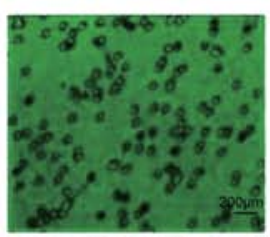

LG+MCGS

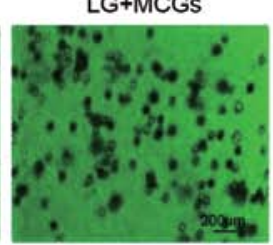

HG+MCGs

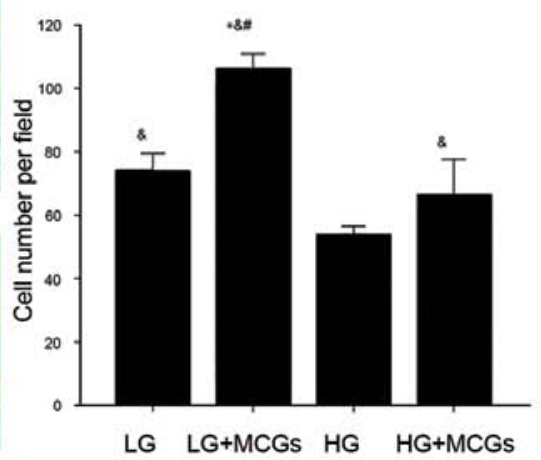

C

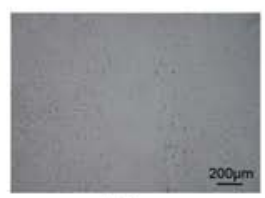

LG

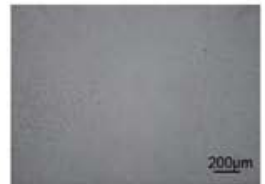

HG

D

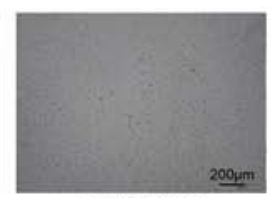

LG+MCGs

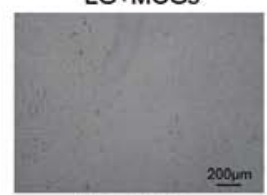

HG+MCGs

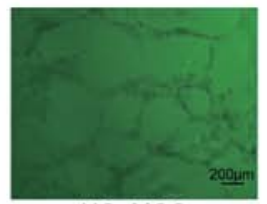

HG+MCGs

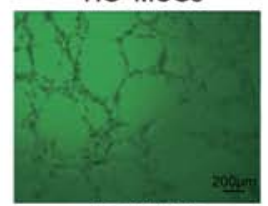

LG+MCGs

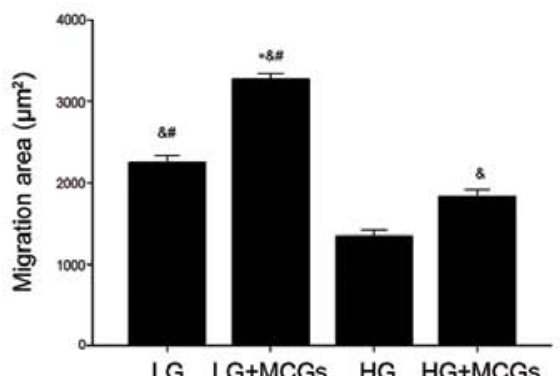

LG LG+MCGs HG HG+MCGs

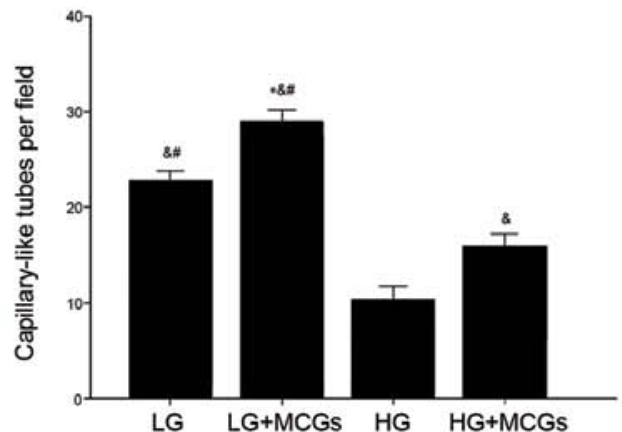

Figure 1. Angiogenic changes under different conditions in vitro. (A) Cell proliferation curve was obtained by alamarBlue ${ }^{\circledR}$ cell viability reagent. (B-D) Representative images of migration across the Transwell membrane, scratch wound healing and capillary-like tube formation, captured under a contrast phase microscope (x200). Values are presented as the means $\pm \mathrm{SE}(\mathrm{n}=3) .{ }^{\&} \mathrm{P}<0.05$ vs. HG; ${ }^{\#} \mathrm{P}<0.05$ vs. HG $+\mathrm{MCGs}$; and ${ }^{*} \mathrm{P}<0.05$ vs. LG. HG, high glucose; LG, low glucose; MCGs, mast cell granules.

tendency continued for up to $48 \mathrm{~h}$ (Fig. 3B). Flk-1 mRNA expression in the MMVECs decreased in a time-dependent manner. Flk-1 mRNA expression in the MMVECs co-cultured with MCGs was significantly higher than that in MMVECs cultured in different concentrations of glucose. Flk-1 mRNA expression in the MMVECs co-cultured with MCGs under hyperglycemic conditions was significantly lower compared to that in MMVECs cultured under low glucose conditions and the tendency continued for up to $48 \mathrm{~h}$ (Fig. 3C).

The addition of either TLCK or TPCK to the co-culture system abolished the effects of MCGs on VEGF, Flt-1 and Flk-1 mRNA expression in the MMVECs cultured in different concentrations of glucose; the effects of TLCK however, were more significant. However, the VEGF, Flt-1 and Flk-1 mRNA expression levels in the MMVECs co-cultured with MCGs following the addition of TLCK were much lower in the absence of MCGs. When the MMVECs were co-cultured with MCGs under hyperglycemic conditions, following the addition of TLCK, VEGF and Flt-1 expression significantly decreased (Fig. 4).

VEGF, Flt-1 and Flk-1 protein expression. VEGF protein expression increased in the MMVECs co-cultured with MCGs with the increase commencing at $2 \mathrm{~h}$ and reaching a peak at $8 \mathrm{~h}$ (Fig. 5) $(\mathrm{P}<0.05)$. Flt-1 protein expression decreased in a timedependent manner in the MMVECs. Flt-1 protein expression in the MMVECs co-cultured with MCGs was significantly higher than that in the MMVECs exposed to different concentrations of glucose. Flt-1 protein expression in the MMVECs cultured under hyperglycemic conditions was significantly lower than that in MMVECs exposed to low glucose levels (Fig. 5B). Flk-1 protein expression decreased in a time-dependent manner 
A

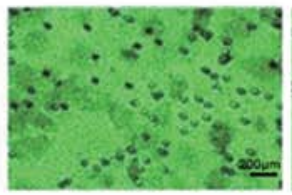

HG+MCGs+TLCK

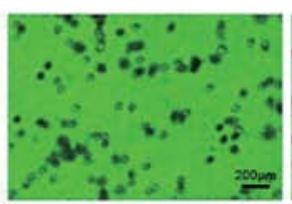

LG+MCGs+TLCK

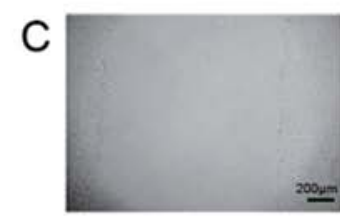

HG+MCGs+TLCK

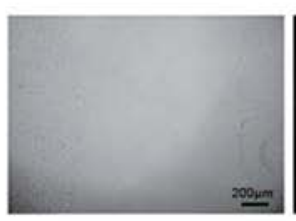

LG+MCGS+TLCK

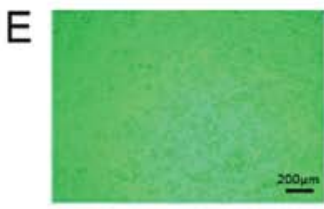

HG+MCGs+TLCK

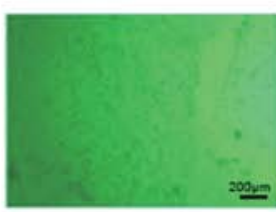

LG+MCGs+TLCK
LG+MCGs+TPCK

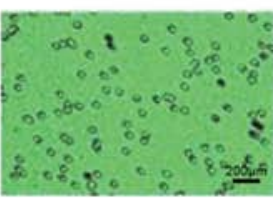

HG+MCGs+TPCK
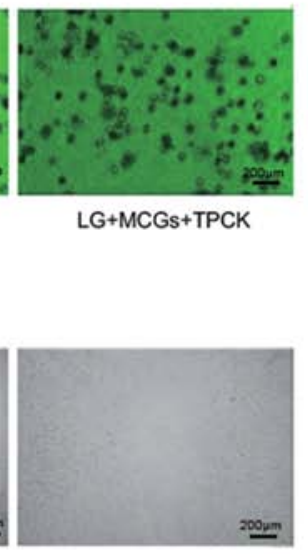

HG+MCGs+TPCK

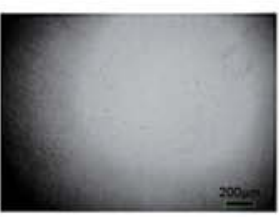

LG+MCGs+TPCK

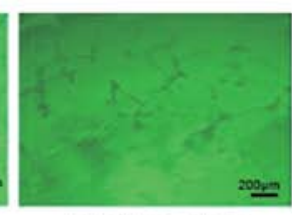

HG+MCGs+TPCK

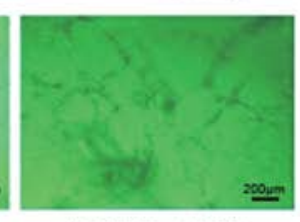

$L G+M C G s+T P C K$
B
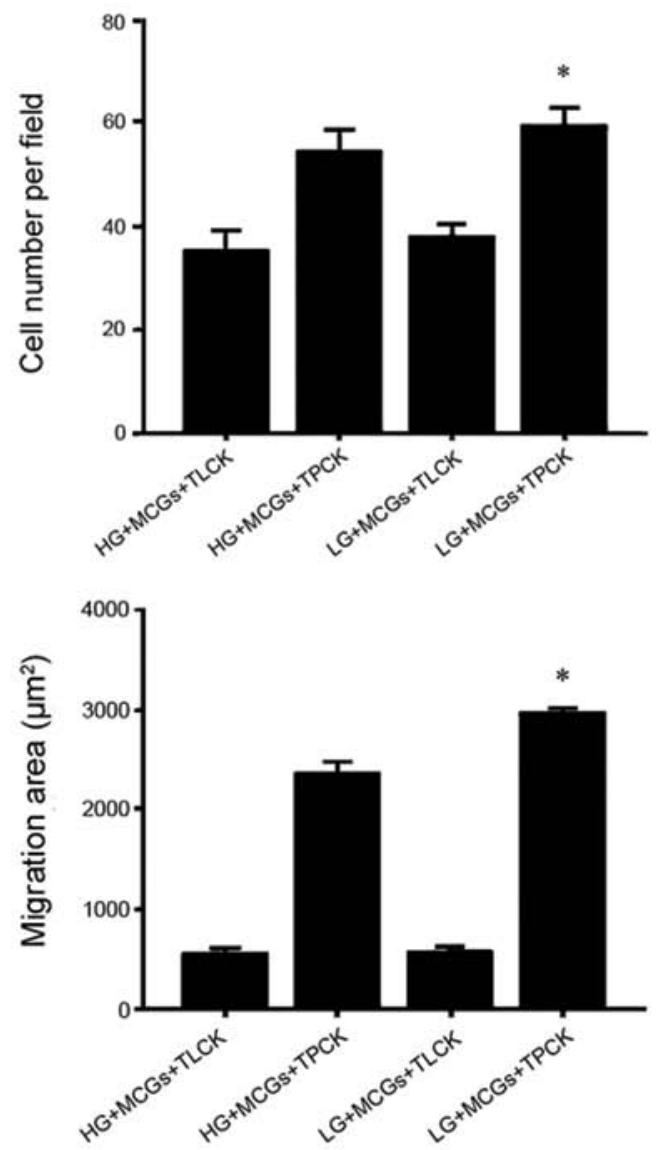

F

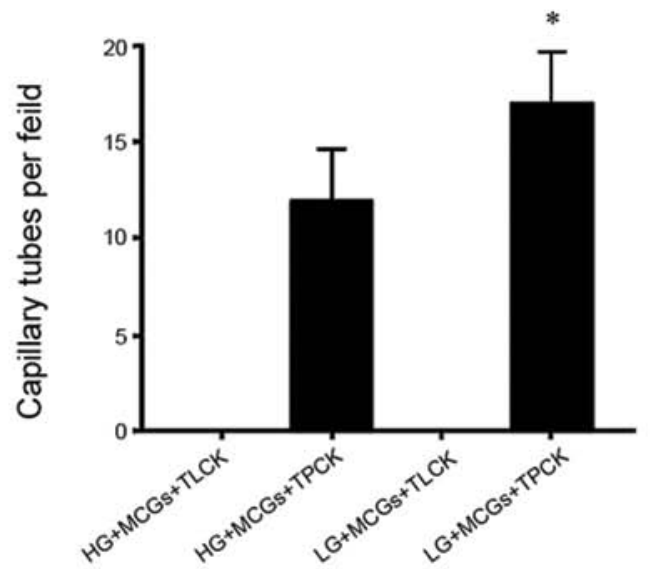

Figure 2. Effect of TLCK or TPCK on proliferation, scratch wound healing and tube formation of myocardial microvascular endothelial cells (MMVECs) cultured in normal and high-glucose medium. Shown are representative (A, C and E) images and (B, D and F) values obtained for cell number, migration area and capillary tube formation, respectively in the cells treated with TLCK or TPCK. ( $\mathrm{P}<0.05$ vs. HG + MCGs + TPCK). TLCK, N-tosyl-L-lysine chloromethyl ketone; TPCK, N-tosyl-L-phenylalanyl chloromethyl ketone; HG, high glucose; LG, low glucose; MCGs, mast cell granules.

in the MMVECs. The Flk-1 protein expression levels in the MMVECs co-cultured with MCGs was significantly higher than that in MMVECs exposed to different glucose concentrations. Flk-1 protein expression in the MMVECs co-cultured with MCGs under hyperglycemic conditions was significantly lower compared to that in MMVECs exposed to low glucose and this tendency continued for up to $48 \mathrm{~h}$ (Fig. 5C).

The addition of either TLCK or TPCK to the co-culture system abolished the effects of MCGs on VEGF, Flt-1 and Flk-1 protein expression in the MMVECs exposed to different concen- trations of glucose; the effects of TLCK though were more significant. The protein expression of VEGF, Flt-1 and Flk-1 following the addition of TLCK to the MMVECs co-cultured with MCGs was much lower than in the MMVECs cultured without MCGs. The protein expression of Flt-1 and Flk-1 in the MMVECs co-cultured with MCGs, after the addition of TLCK under low glucose conditions, was significantly higher than that in the MMVECs cultured without MCGs. However, it is noteworthy that this phenomenon was not observed in the MMVECs cultured under hyperglycemic conditions (Fig. 6). 

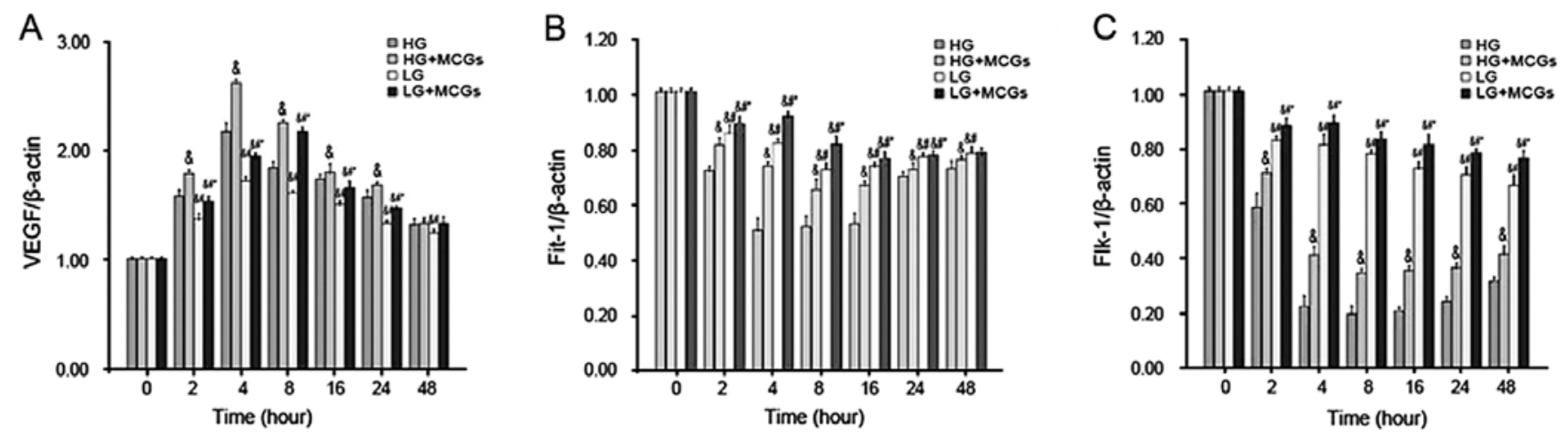

Figure 3. Vascular endothelial growth factor (VEGF), fms-like tyrosine kinase-1 (Flt-1) and fetal liver kinase-1 (Flk-1) mRNA expression in myocardial microvascular endothelial cells (MMVECs). (A) VEGF expression increased significantly in MMVECs cultured with MCGs at $2 \mathrm{~h}$, VEGF expression reached at its peak point at $4 \mathrm{~h}$ in the HG group, and at $8 \mathrm{~h}$ in the LG group. Even in the presence of MCGs, VEGF expression in the LG group was significantly lower than that in the HG group $(\mathrm{P}<0.05)$. (B) Flt-1 expression decreased significantly in the MMVECs at $2 \mathrm{~h}(\mathrm{P}<0.05)$, and reached at its low point at $4 \mathrm{~h}$ in the $\mathrm{HG}$ group, and at $8 \mathrm{~h}$ in the LG group. (C) Flt-1 expression was significantly different between the HG and LG group $(\mathrm{P}<0.05)$. Flt-1 expression was significantly higher in the presence of MCGs. Flk-1 expression decreased significantly in the HG group at $2 \mathrm{~h}$, and reached its low point at $8 \mathrm{~h}$. Even in the presence of MCGs, Flk-1 expression in the HG group was significantly lower than that in the $\mathrm{LG}$ group $(\mathrm{P}<0.05)$. ${ }^{\circledR} \mathrm{P}<0.05$ vs. HG; ${ }^{*} \mathrm{P}<0.05$ vs. HG + MCGs; and ${ }^{*} \mathrm{P}<0.05$ vs. LG. HG, high glucose; LG, low glucose; MCGs, mast cell granules.
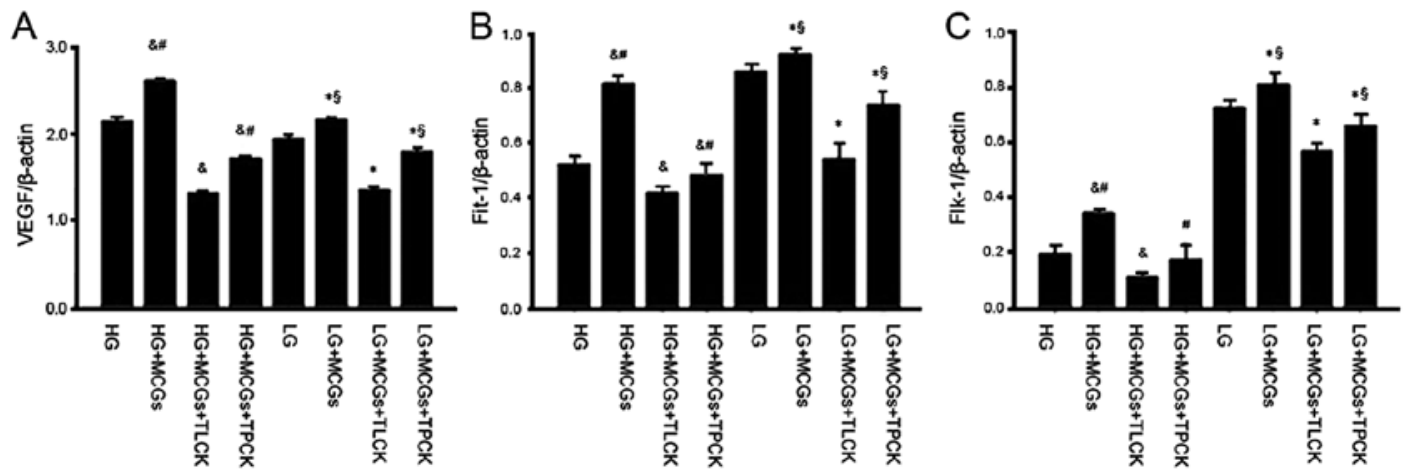

Figure 4. Vascular endothelial growth factor (VEGF), fms-like tyrosine kinase-1 (Flt-1) and fetal liver kinase-1 (Flk-1) mRNA expression in myocardial microvascular endothelial cells (MMVECs) cultured in the presence of TLCK or TPCK. The mRNA expression of VEGF, Flt-1 and Flk-1 decreased significantly in the presence of TLCK or TPCK, particularly in the presence of TLCK, though the mRNA expression of VEGF in the LG group was not significantly different from that in the HG group; however, the expression of Flt-1 and FLK-1 in the LG group was significantly higher than that in the HG group $(\mathrm{P}<0.05)$. ${ }^{\mathrm{P}}<0.05$ vs. HG; ${ }^{\#} \mathrm{P}<0.05$ vs. HG + MCGs + TLCK; "P<0.05 vs. LG; and ${ }^{8} \mathrm{LG}+$ MCGs + TLCK). TLCK, N-tosyl-L-lysine chloromethyl ketone; TPCK, N-tosyl-Lphenylalanyl chloromethyl ketone; HG, high glucose; LG, low glucose; MCGs, mast cell granules.

\section{Discussion}

Angiogenesis is tightly regulated by pro- and anti-angiogenic factors. In tumor models, MCs have been shown to play a decisive role in inducing the angiogenic switch that precedes malignant transformation. MCs are a rich source of several potent angiogenic factors, including VEGF, bFGF, transforming growth factor (TGF)- $\beta$, tumor necrosis factor (TNF)- $\alpha$ and interleukin (IL)-8 $(20,21)$. In our study, we found that a close correlation exists between angiogenesis and various growth factors in MMVECs co-cultued with MCGs. In our co-culture system, MCGs increased the migration and lumen formation in MMVECs, as well as the expression of VEGF, Flt-1 and Flk-1.

VEGF, as a major regulator of angiogenesis, binds and activates 2 tyrosine kinase receptors, Flt- 1 and Flk-1. These receptors regulate physiological, as well as pathological angiogenesis. Flk-1 has strong tyrosine kinase activity, and transduces the major signals for angiogenesis. Flk-1 is a direct signal transducer for pathological angiogenesis, including cancer and diabetic retinopathy. Although the affinity of Flt-1 to VEGF is more than 10-fold that of Flk-1 (22), Flk-1 plays an important role in proliferation, migration and the survival process. Thus, Flk-1 and its signaling appear to be critical targets for the suppression of these diseases. It has been established that high glucose levels are the direct cause of capillary vessel damage in diabetes mellitus $(23,24)$. Sasso et al $(25)$ found that the expression of VEGF in individuals with chronic coronary artery disease and diabetes mellitus was significantly higher compared to individuals who did not suffer from diabetes mellitus; however, in our study, even in the presence of MCGs exposed to high glucose, this tendency still existed. The expression of VEGF in the MMVECs exposed to high glucose was significantly higher than that in the MMVECs 
A

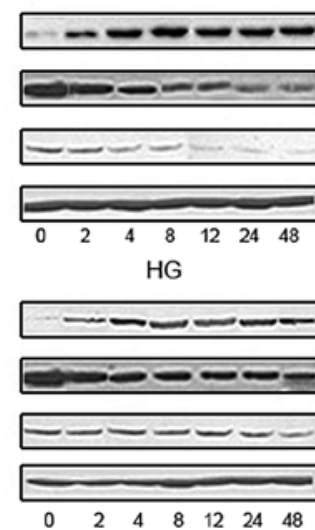

LG

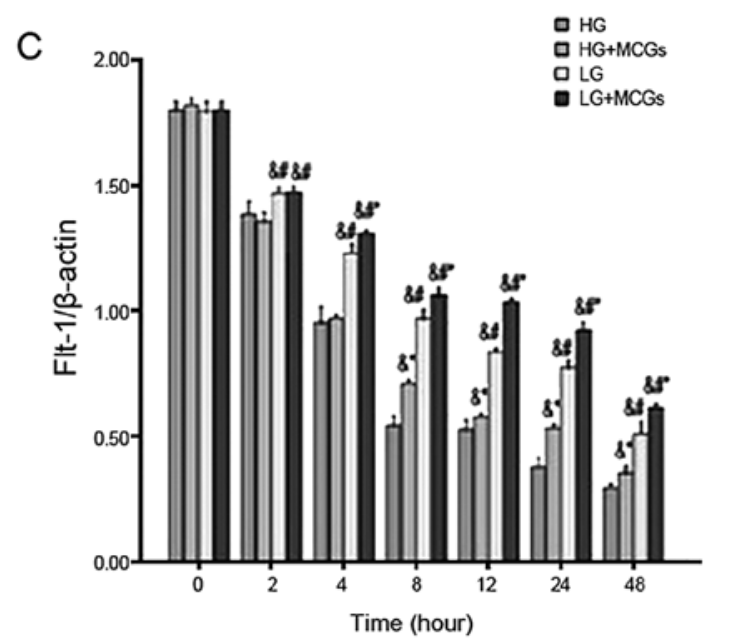

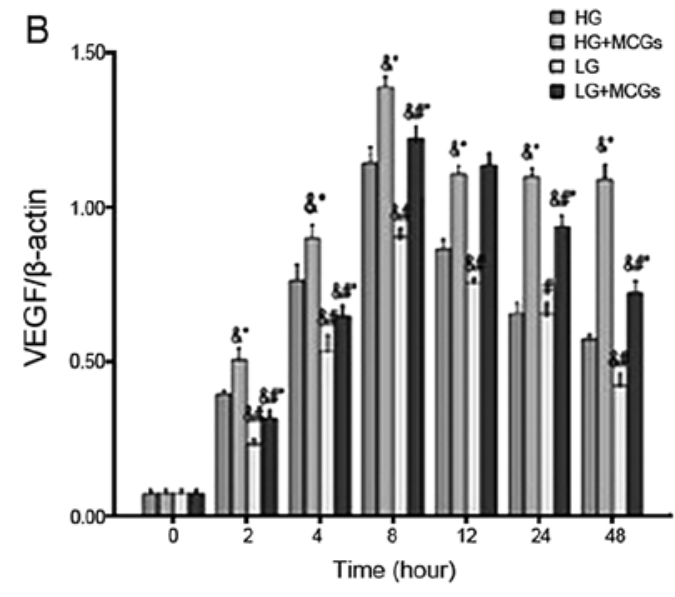

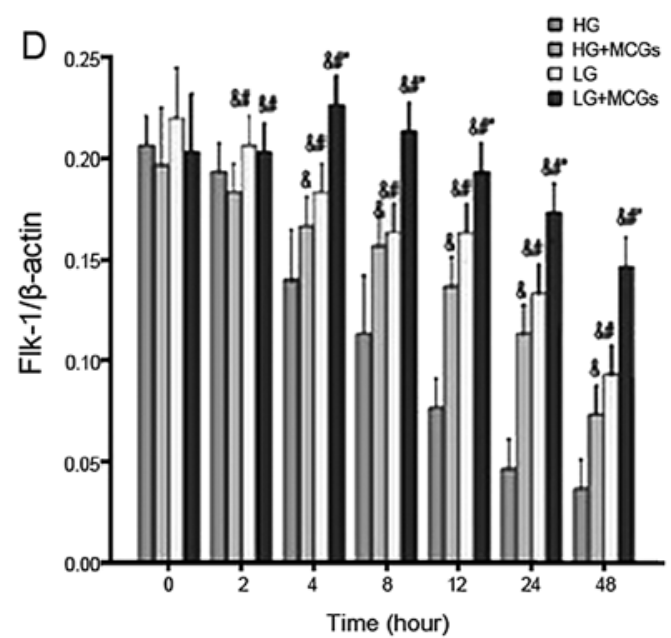

Figure 5. Vascular endothelial growth factor (VEGF), fms-like tyrosine kinase-1 (Flt-1) and fetal liver kinase-1 (Flk-1) protein expression in myocardial microvascular endothelial cells (MMVECs) cultured under different conditions. (A) Western blot analysis of VEGF, Flt-1 and Flk-1 protein expression in MMVECs cultured under high or low glucose conditions without or without MCGs. (B) VEGF expression increased significantly in MMVECs cultured with MCGs at $2 \mathrm{~h}$, and reached at its peak point at $8 \mathrm{~h}$. In the presence of MCGs, VEGF expression was significantly higher than that in absence of MCGs (P<0.05). (C) Flt-1 expression decreased significantly in the MMVECs at $2 \mathrm{~h}$. The expression of Flt-1 in the LG group was significantly high than that in the HG group and Flt-1 expression was significantly higher in presence of MCGs. (D) Flk-1 significantly decreased significantly at $2 \mathrm{~h}$. Even in the presence of MCGs, Flk-1 significantly in the HG group was significantly lower than that in the LG group. ( ${ }^{\circledR} \mathrm{P}<0.05$ vs. HG; ${ }^{*} \mathrm{P}<0.05$ vs. HG + MCGs; and ${ }^{*} \mathrm{P}<0.05$ vs. LG). HG, high glucose; LG, low glucose; MCGs, mast cell granules.
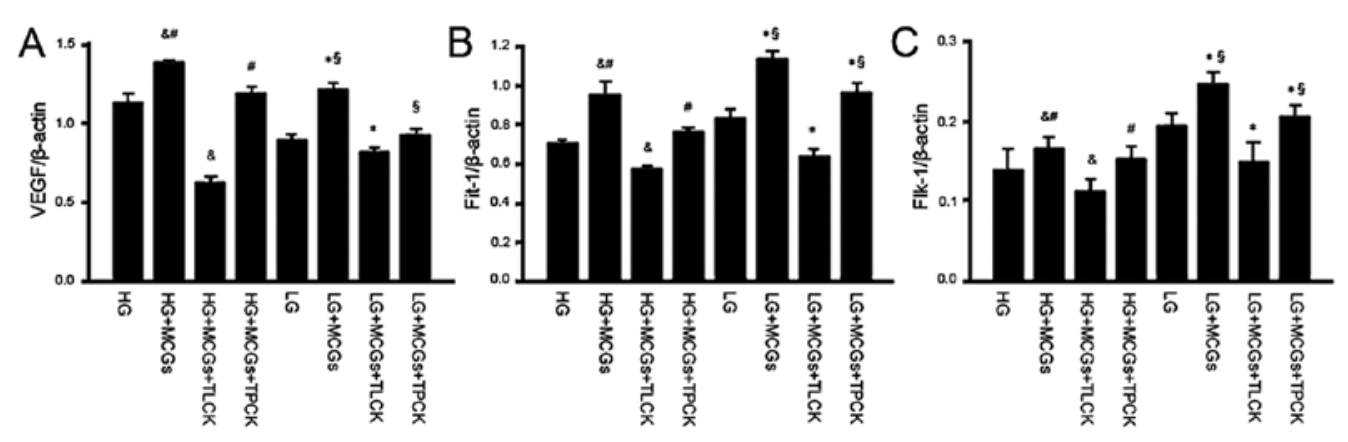

Figure 6. Vascular endothelial growth factor (VEGF), fms-like tyrosine kinase-1 (Flt-1) and fetal liver kinase-1 (Flk-1) protein expression in myocardial microvascular endothelial cells (MMVECs) in the presence of TLCK or TPCK. The expression of VEGF, Flt-1 and Flk-1 decreased significantly in the presence of TLCK. The expression of VEGF, Flt-1 and FLK-1 in the LG group was significantly higher than that in the HG group $(\mathrm{P}<0.05)$. ${ }^{\text {}} \mathrm{P}<0.05$ vs. HG; ${ }^{\text {" }} \mathrm{P}<0.05$ vs. HG + MCGs + TLCK; ${ }^{*}$ P $<0.05$ vs. LG; and ${ }^{\&} \mathrm{LG}+$ MCGs + TLCK. TLCK, N-tosyl-L-lysine chloromethyl ketone; TPCK, N-tosyl-L-phenylalanyl chloromethyl ketone; HG, high glucose; LG, low glucose; MCGs, mast cell granules.

exposed to low glucose; the expression of Flt-1, Flk-1 and their downstream signals was significantly suppressed. Chou et al found that the expression of VEGF, Flt-1 and Flk-1 were signif- icantly decreased in heart tissues but were increased 2-fold in the retina and kidney in diabetes mellitus patients (25). In the current study, we found that capillary-like tube formation 
and the migration of MMVECs cultured under low glucose conditions increased compared to MMVECs cultured under high glucose conditions; however, Flt-1 and Flk-1 expression decreased, particularly Flk-1 expression. The reason that capillary-like tube formation and the migration of MMVECs cultured under low glucose conditions increased significantly, compared to the MMVECs cultured under high glucose conditions, even in the presence of MCGs, may be the fact that the expression of VEGF increased. However, the expression of the receptors decreased and the expression of Ang-2 increased, all these factors likely affecting the angiogenic ability of MMVECs in high glucose. These results demonstrate that high glucose levels are an important factor for the angiogenic ability of MMVECs in vitro. We also found that the duration of culture had a pronounced effect on MMVEC growth under high glucose conditions. Su found that the number of MMVECs cultured in high glucose for 24-96 $\mathrm{h}$ increased in the transcriptionally silent period and decreased in the DNA synthesis period (26). Abe found that the number of retina cells cultured in high glucose decreased by $78 \%$ after 10 days (27). We also found that the expression of VEGF and its receptors in MMVECs significantly decreased as the culture duration increased.

Tryptase and chymase are the predominant proteases present in MCGs; the amount of these proteases is $20-50 \%$ in MCs. Tryptase directly added to dermal microvascular endothelial cells caused a significant augmentation of capillary growth, which was suppressed by specific tryptase inhibitors. Tryptase also directly induced the cell proliferation of human dermal microvascular endothelial cells (HDMECs) in a concentration-dependent manner (28). MCs act at sites of new vessel formation by secreting tryptase, which then functions as a potent and previously unrecognized angiogenic factor (28). In our study, we found that a close correlation existed between MMVECs and MCGs and angiogenesis. In our co-culture system, MCGs increased migration and lumen formation in MMVECs, but the application of TLCK or TPCK significantly attenuated angiogenesis, in spite of the glucose concentration; TLCK was particularly effective in this respect. We also found that capillary-like tube formation and the migration of MMVECs co-cultured with MCGs, when exposed to TLCK or TPCK, significantly decreased. It seems that MCGs contain or secrete many of the angiogenic and anti-angiogenic factors. When the main mediators of angiogenesis, tryptase and chymase, were inhibited, anti-angiogenic factors in MCGs, such as endothelin-1 (ET-1), had a significant anti-angiogenic effect.

Liu et al found that MCs contribute to obesity and diabetes. Mechanism studies have revealed that IL-6 and interferon (IFN)- $\gamma$ in MCs contribute to WAT and muscle angiogenesis (5). However, this result was not so different from our findings. The reason may be that: i) our study focused on MMVECs co-cultured with MCGs in vitro; ii) diabetes mellitus is a chronic process, while cell culture studies are necessarily conducted over a much shorter time period; it may well be a completely different pathophysiological process; and iii) the angiogenic process of MCs isolated from animals is likely to be complex and perhaps differs from what occurs in other cell types in vitro.
In conclusion, our results demonstrate unequivocally that tryptase is the main angiogenic mediator in MCGs. High glucose levels have a profound effect on angiogenesis; this effect may be more pronounced than the effects of MCGs on angiogenesis.

\section{Acknowledgements}

The present study was supported by an Institutional Research Grant (to M.W.) and the Shanghai PuJiang Program [PJ (2008) 00586 to Q.Z.].

\section{References}

1. Robbie-Ryan M and Brown M: The role of mast cells in allergy and autoimmunity. Curr Opin Immunol 14: 728-733, 2002.

2. Sun J, Sukhova GK, Wolters PJ, et al: Mast cells promote atherosclerosis by releasing proinflammatory cytokines. Nat Med 13: 719-724, 2007.

3. Sun J, Sukhova GK, Yang M, et al: Mast cells modulate the pathogenesis of elastase-induced abdominal aortic aneurysms in mice. J Clin Invest 117: 3359-3368, 2007.

4. Coussens LM, Raymond WW, Bergers G, et al: Inflammatory mast cells up-regulate angiogenesis during squamous epithelial carcinogenesis. Genes Dev 13: 1382-1397, 1999.

5. Liu J, Divoux A, Sun J, et al: Genetic deficiency and pharmacological stabilization of mast cells reduce diet-induced obesity and diabetes in mice. Nat Med 15: 940-945, 2009.

6. Pang C, Gao Z, Yin J, Zhang J, Jia W and Ye J: Macrophage infiltration into adipose tissue may promote angiogenesis for adipose tissue remodeling in obesity. Am J Physiol Endocrinol Metab 295: E313-E322, 2008.

7. Kintscher U, Hartge M, Hess K, et al: T-lymphocyte infiltration in visceral adipose tissue: a primary event in adipose tissue inflammation and the development of obesity-mediated insulin resistance. Arterioscler Thromb Vasc Biol 28: 1304-1310, 2008.

8. Rupnick MA, Panigrahy D, Zhang CY, et al: Adipose tissue mass can be regulated through the vasculature. Proc Natl Acad Sci USA 99: 10730-10735, 2002.

9. Sato K, Tsuchihara K, Fujii S, et al: Autophagy is activated in colorectal cancer cells and contributes to the tolerance to nutrient deprivation. Cancer Res 67: 9677-9684, 2007.

10. Holash J, Maisonpierre PC, Compton D, et al: Vessel cooption, regression, and growth in tumors mediated by angiopoietins and VEGF. Science 284: 1994-1998, 1999.

11. Holash J, Wiegand SJ and Yancopoulos GD: New model of tumor angiogenesis: dynamic balance between vessel regression and growth mediated by angiopoietins and VEGF. Oncogene 18: 5356-5362, 1999.

12. Zygalaki E, Kaklamanis L, Nikolaou NI, et al: Expression profile of total VEGF, VEGF splice variants and VEGF receptors in the myocardium and arterial vasculature of diabetic and nondiabetic patients with coronary artery disease. Clin Biochem 41: 82-87, 2008.

13. Chen JX and Stinnett A: Disruption of Ang-1/Tie-2 signaling contributes to the impaired myocardial vascular maturation and angiogenesis in type II diabetic mice. Arterioscler Thromb Vasc Biol 28: 1606-1613, 2008.

14. Ning YX, Wang XH, Jin HM, Zhao FD and Yin LH: Study on the culture of rat myocardium microvascular endothelial cells and microarray analysis. Zhongguo Bing Li Sheng Li Za Zhi 21: 2295-2300, 2005 (In Chinese).

15. Kokkonen JO and Kovanen PT: Low density lipoprotein degradation by rat mast cells. Demonstration of extracellular proteolysis caused by mast cell granules. J Biol Chem 260: 14756-14763, 1985.

16. Kokkonen JO and Kovanen PT: Accumulation of low density lipoproteins in stimulated rat serosal mast cells during recovery from degranulation. J Lipid Res 30: 1341-1348, 1989.

17. He S, Peng Q and Walls AF: Potent induction of a neutrophil and eosinophil-rich infiltrate in vivo by human mast cell tryptase: selective enhancement of eosinophil recruitment by histamine. J Immunol 159: 6216-6225, 1997. 
18. Ettenson DS and Gotlieb AI: Centrosomes, microtubules, and microfilaments in the reendothelialization and remodeling of double-sided in vitro wounds. Lab Invest 66: 722-733, 1992.

19. Pollman MJ, Naumovski L and Gibbons GH: Endothelial cell apoptosis in capillary network remodeling. J Cell Physiol 178: 359-370, 1999

20. Qu Z, Liebler JM, Powers MR, et al: Mast cells are a major source of basic fibroblast growth factor in chronic inflammation and cutaneous hemangioma. Am J Pathol 147: 564-573, 1995.

21. Ribatti D, Crivellato E, Candussio L, et al: Mast cells and their secretory granules are angiogenic in the chick embryo chorioallantoic membrane. Clin Exp Allergy 31: 602-608, 2001.

22. Shibuya M: Structure and dual function of vascular endothelial growth factor receptor-1 (Flt-1). Int J Biochem Cell Biol 33: 409-420, 2001

23. Klein R: Hyperglycemia and microvascular and macrovascular disease in diabetes. Diabetes Care 18: 258-268, 1995.

24. Chou E, Suzuma I, Way KJ, et al: Decreased cardiac expression of vascular endothelial growth factor and its receptors in insulinresistant and diabetic states: a possible explanation for impaired collateral formation in cardiac tissue. Circulation 105: 373-379, 2002.
25. Sasso FC, Torella D, Carbonara O, et al: Increased vascular endothelial growth factor expression but impaired vascular endothelial growth factor receptor signaling in the myocardium of type 2 diabetic patients with chronic coronary heart disease. J Am Coll Cardiol 46: 827-834, 2005.

26. Su Y, Liu XM, Sun YM, et al: Endothelial dysfunction in impaired fasting glycemia, impaired glucose tolerance, and type 2 diabetes mellitus. Am J Cardiol 102: 497-478, 2008.

27. Abe T, Yoshida M, Yoshioka Y, et al: Iris pigment epithelial cell transplantation for degenerative retinal diseases. Prog Retin Eye Res 26: 302-321, 2007.

28. Blair RJ, Meng H, Marchese MJ, et al: Human mast cells stimulate vascular tube formation. Tryptase is a novel, potent angiogenic factor. J Clin Invest 99: 2691-2700, 1997. 